(CCP3) and rheumatoid factor (RF)] data were collected at baseline. Regression analyses, Kaplan-Meier analysis and Log-Rank test were performed.

Results: US synovitis was detected in $\geq 1$ longitudinal US scan in 69/186 (37.1\%) at-risk individuals (median time to first developing US synovitis: 53 weeks, IQR 27.0-105.8; median number of joints with US synovitis: 2.0, IQR 1.0-2.0). As shown in Table 1, only anti-CCP3 Ab were significantly associated with development of US sub-clinical synovitis in the multi-variable analysis while borderline results were observed with age.

Table 1. Regression analyses for the development of US synovitis.

\begin{tabular}{lllll}
\hline & \multicolumn{2}{l}{ Univariable analysis } & Multivariable & analysis \\
\hline & OR $(95 \% \mathrm{Cl})$ & p-value & OR $(95 \% \mathrm{Cl})$ & p-value \\
\hline Gender (male) & $1.02(0.52-2.02)$ & 0.95 & $/$ & $/$ \\
Age & $\mathbf{1 . 0 3 ( 1 . 0 1 - 1 . 0 6 )}$ & $<0.01$ & $1.03(1.00-1.06)$ & 0.03 \\
Tenderness in the hands & $0.86(0.46-1.61)$ & 0.64 & $/$ & $/$ \\
EMS & $1.60(0.87-2.95)$ & 0.13 & $/$ & $/$ \\
Anti-CCP2 Ab (high titre) & $\mathbf{2 . 7 9 ( 1 . 3 7 - 5 . 6 7 )}$ & $<0.01$ & $1.20(0.50-2.89)$ & 0.69 \\
Anti-CCP3+ & $\mathbf{4 . 4 4 ( 2 . 2 8 - 8 . 6 6 )}$ & $<0.01$ & $\mathbf{3 . 3 0}(1.39-7.89)$ & $<0.01$ \\
RF+ & $\mathbf{2 . 9 6 ( 0 . 4 6 - 1 . 6 1 )}$ & $\mathbf{0 . 0 1}$ & $1.45(0.68-3.11)$ & 0.33 \\
\hline
\end{tabular}

$\mathrm{CCP} 2+$ individuals with positive anti-CCP3 $\mathrm{Ab}$ show a significantly reduced sub-clinical synovitis-free survival rate compared with individuals with negative anti-CCP3 Ab (Figure 1). At 1- and 2-year follow-up, respectively $23.3 \%$ and $38.3 \%$ of individuals with dual CCP2/CCP3 positivity developed sub-clinical synovitis on longitudinal scans, compared with $8.4 \%$ and $13.3 \%$ of $\mathrm{CCP} 2+$ individuals with negative anti-CCP3 Ab $(p=0.01)$ (Figure 1a).

a)

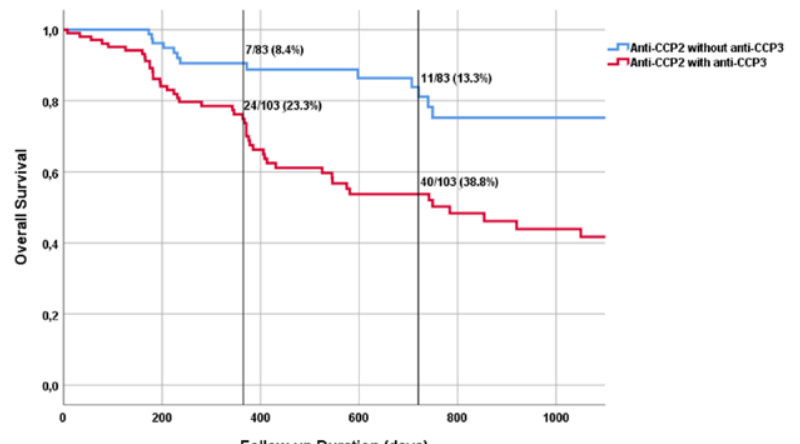

b)

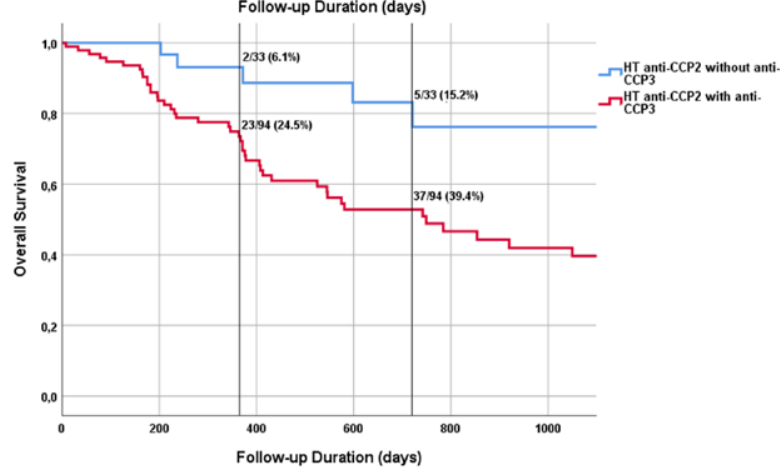

Figure 1. Kaplan-Meier analysis shows US sub-clinical synovitis free survival time in CCP2+ at-risk individuals.

Similar results were observed in the subgroup of high level CCP2+ individuals. At 1- and 2 -year follow-up, respectively $24.5 \%$ and $39.4 \%$ of high level CPP2/antiCCP3+, but only $6.1 \%$ and $15.2 \%$ of CCP2+ individuals with negative anti-CCP3 developed sub-clinical synovitis on longitudinal scans $(p<0.01)$ (Figure $1 b)$.

Conclusion: In anti-CCP2+ at-risk individuals with MSK symptoms, anti-CCP3 antibodies improve prediction of imminent development of subclinical joint inflammation. This may represent the critical time-point for interventions to prevent the onset of joint disease. This is also a unique population for investigating the drivers of joint involvement in the development of RA.

REFERENCES:

[1] Duquenne L, et al. The Role of Ultrasound Across the Inflammatory Arthritis Continuum: Focus on "At-Risk" Individuals. 2020

[2] D'Agostino MA, et al. Scoring ultrasound synovitis in rheumatoid arthritis: a

EULAR-OMERACT ultrasound taskforce - Part 1: definition and develop-

ment of a standardised, consensus-based scoring system. 2017.

Disclosure of Interests: Andrea Di Matteo Grant/research support from: This study was conducted while Andrea Di Matteo was an ARTICULUM Fellow., Kulveer Mankia Speakers bureau: KM reports personal fees from Abbvie, UCB and Eli Lilly (all $<\$ 10.000$ ), outside the submitted work., Grant/research support from:
Research grants from BMS, Eli Lilly (all $<\$ 10.000)$, Laurence Duquenne: None declared, Edoardo Cipolletta: None declared, Jacqueline Nam: None declared, Leticia Garcia-Montoya: None declared, Richard Wakefield Speakers bureau: RJW has received honoraria from Abbvie, Novartis and GE for ultrasound related educational activities (all $<\$ 10.000$ )., Michael Mahler Employee of: MM is employee of Inova Diagnostics, commercializing CCP3, Paul Emery Speakers bureau: PE reports consultant fees from BMS, AbbVie, Gilead, Galapagos Lilly, MSD, Pfizer, Novartis, Roche, and Samsung outside the submitted work (all $<\$ 10.000)$., Grant/research support from: He also reports research grants from UCB, AbbVie, Lilly, Novartis, BMS, Pfizer, MSD and Roche, outside the submitted work. PE is National Institute for Health Research (NIHR) Biomedical Research Centre (BRC) director and BRC funds supported this work. Leticia Garcia-Montoya and Laurence Duquenne are NIHR BRC fellows. DOI: 10.1136/annrheumdis-2021-eular.2290

\section{POS0465 DIFFERENT CLINICAL RELEVANCE OF ANTI- CITRULLINATED PROTEINS ANTIBODIES IN RA PATIENTS}

A. Avdeeva ${ }^{1}$, M. Cherkasova ${ }^{2}$, E. Nasonov ${ }^{2}{ }^{1}$ V.A. Nasonova Research Institute of Rheumatology, Early Arthritis Laboratory, Moscow, Russian Federation; ${ }^{2}$ V.A. Nasonova Research Institute of Rheumatology, Laboratory of Immunology and Molecular Biology Rheumatic Diseases, Moscow, Russian Federation

Background: Anti-citrullinated proteins antibodies (ACPA) are a broad group of antibodies, including antibodies to citrullinated fibrinogen, antibodies to cyclic citrullinated peptide (anti-CCP), antibodies to modified citrullinated vimentin (antiMCV), antibodies to citrullinated $\alpha$-enolase.

Objectives: To find a potential relationship between ACPA and disease activity, bone destruction, and ACPAs responses to various therapeutic regimens.

Methods: The study included 232 patients (pts) with rheumatoid arthritis (RA) 90 pts (74 women, Me;IQR age 53.0 (38.0-58.5) years had early RA, with mean disease duration 5.0 (4.0-9.0) months, DAS 285.3 (4.4-6.1)); 142 pts had advanced stage of the disease (123 women, median age 51.0 (43.0-60.0) years, disease duration 56.0 (24.0-96.0) months, DAS 286.2 (5.5-6.8)). Pts with early RA received methotrexate (MTX) subcutaneously at average $17.5 \mathrm{mg}$ dose once weekly. Pts with advanced RA received the following anti-B-cell therapy: 34 pts - rituximab (RTX); 20 pts - RTX biosimilar; 43 pts - tocilizumab (TCZ) in combination with conventional DMARDs. Serum anti-CCP and anti-MCV concentrations were measured using ELISA

Results: 77 (85.6\%) pts with early RA were high positive for anti-CCP, and 29 $(70.7 \%)$ pts - high positive for anti-MCV. A positive correlation was found between anti-MCV and DAS 28 ( $r=0.4, p=0.04)$. As for advanced RA, $78(80.4 \%)$ pts were high positive for anti-CCP, and $70(79.5 \%)$ - for anti-MCV. There was a positive correlation between anti-MCV concentration and SDAI ( $r=0.4, p=0.02)$, as well as CDAI $(r=0.4, p=0.02)$. No significant correlations were found between the antiCCP levels and activity indices, anti-CCP and acute-phase parameters in both early and advanced RA groups. Higher total Sharp scores (96.5 (65.0-122.0)) were found in pts high positive for anti-MCV $(n=79)$, compared to low-positive/ negative (57.0 (31.0-88.0), respectively, $(n=27, p<0.05)$. anti-MCV levels dropped significantly in pts on RTX and TCZ therapy at weeks 12 and 24 after initiation of treatment, while high anti-CCP concentration persisted throughout the treatment (Table 1)

Table 1.

\begin{tabular}{|l|ll|}
\hline Indicators & Anti-CCP, U/ml & Anti-MCV, U/ml \\
\hline RTX group, $n=34$ & & \\
- baseline & $100,0(37,9-100,0)$ & $559,4(139,2-1000,0)$ \\
- 12 weeks & $100,0(26,1-100,0)$ & $295,9(74,0-962,7)^{1}$ \\
- 24 weeks & $100,0(29,0-100,0)$ & $194,7(58,3-844,8)^{1}$ \\
\hline RTX biosimilar group, $n=20$ & $112,7(18,3-264,8)$ & $392,6(75,7-1000,0)$ \\
- baseline & $71,7(12,4-161,6)$ & $210,5(40,3-940,6)^{1}$ \\
- 12 weeks & $61,3(13,12-129,4)$ & $153,8(43,1-702,8)$ \\
- 24 weeks & & \\
TCZ group, $n=43$ & $354,8(67,9-500,0)$ & $762,3(106,9-2393,1)$ \\
- baseline & $500,0(84,4-500,0)^{1}$ & $266,6(85,8-927,0)^{1}$ \\
- 8 weeks & $355,8(39,8-500,0)$ & $135,7(27,0-662,1)^{1}$ \\
- 24 weeks & & \\
Note: ${ }^{1} p<0,05$ vs baseline & &
\end{tabular}

Conclusion: anti-MCV levels correlated with inflammatory activity and development of bone destruction, and were decreasing in pts on treatment. Anti-CCP was less responsive, showed minor changes during treatment, therefore its' thorough monitoring was not feasible.

Disclosure of Interests: None declared

DOI: 10.1136/annrheumdis-2021-eular.2320 